\section{Aurora A kinase as a target for therapy in TCF3-HLF rearranged acute lymphoblastic leukemia}

B-cell acute lymphoblastic leukemia (B-ALL) harboring the $t(17 ; 19)(q 22 ; p 13)$ is a rare subtype of leukemia with a dismal prognosis. ${ }^{1}$ This translocation produces an aberrant TCF3-HLF fusion with distinct gene expression profiles and drug sensitivity. Recent studies have shown that this subtype of B-ALL may be amenable to therapies inhibiting BCL2 and the pre-B cell receptor through inhibition of SRC family kinases. ${ }^{2-4}$ Using RNA sequencing (RNA-seq) in combination with ex vivo drug sensitivity analyses we identified an overexpression pattern of Aurora A kinase (AURKA) in five of five TCF3-HLF ALL samples. This finding further translated to enhanced sensitivity to inhibition by one of the AURKA inhibitors alisertib. Our studies suggest a molecular susceptibility of TCF3-HLF ALL to alisertib providing support to pursue further clinical testing within this rare and lethal subtype of ALL.

The $t(17 ; 19)(q 22 ; p 13)$ rearrangement encodes for a chimeric transcription factor consisting of type 1 rearrangements with a fusion of exon 16 of TCF3 with exon 4 of $H L F$; and type 2 rearrangements aligning with a fusion of exon 15 of TCF3 with exon 4 of $H L F .{ }^{5}$ Because of its rarity and poor prognosis, there are few established recommended therapies. Patients who achieve remission with no evidence of disease historically recur and die of disease even with stem cell transplant as consolidation therapy. ${ }^{6}$ Recent advances using immunologic therapies has shown some promise with durable responses targeting expression of CD19 by B-cell leukemia, ${ }^{7}$ however not all patients remained in remission and not all may be eligible for immunologic therapies. Therefore, novel therapies remain essential to improve outcomes. We developed an in vitro assay using a panel of small-molecule inhibitors to identify patient specific targeted therapies and employed this assay on the diagnostic marrow sample of a patient who presented to our institution with TCF3-HLF ALL. ${ }^{2}$ We observed in vitro sensitivity to the ABL/SRC inhibitor dasatinib with some clinical benefit. ${ }^{2}$ Meanwhile, other studies identified targeting the BCL2 family of proteins with venetoclax as a therapeutic potential. ${ }^{3,4}$ We thus sought to validate and identify other novel targets across an independent set of samples. We partnered with the Children's Oncology Group (COG) and St. Jude Children's Research Hospital to obtain four separate patient samples combined with our sample which were xenografted and expanded for in vitro as well as in vivo experiments. All clinical samples were obtained with informed consent with approval by the Institutional Review Board of Oregon Health \& Science University, the Children's Oncology Group, and St. Jude Children's Research Hospital. All murine studies were approved by the OHSU Institutional Animal Care and Use Committee (IACUC, protocol \#2358).

Standard reverse transcription polymerase chain reaction (RT-PCR), Sanger sequencing and immunoblot analysis of the TCF3-HLF fusion identified three samples with a type 1 translocation (10-199, 3.316, and 3.332) with variable insertions corresponding to different protein length, while two others carried a type 2 translocation $(3310,3324)$ (Online Supplementary Figure S1A). We then undertook next-generation sequencing studies with these samples compared to 35 B-ALL samples (Online Supplementary Figure $S 1 B$ and $C$ ). Using whole-exome sequencing within our ALL cohort, and specifically focusing on variations found in pathways in B-cell ALL, ${ }^{8}$ we found that three of the five samples carried an IKZF1 variant of unknown clinical significance (IKZF1 I125V; Online Supplementary Figure $S 1 B)$. Further, two of the five samples carried KRAS mutations (KRAS G12V/S) as previously described. ${ }^{9}$ Finally, RNA-seq analysis of the top 1.000 most variably expressed genes across the cohort revealed gene expression signatures that clustered strongly with TCF3-HLF ALL (Online Supplementary Figure $S 1 C$ ), suggesting relatively uniform gene expression within these five Type 1 and Type 2 TCF3-HLF leukemias.

Implementation of our small-molecule kinase inhibitor pane $1^{10}$ on 69 ALL samples revealed some consistent patterns of responses in TCF3-HLF ALL including sensitivity to the class of aurora kinase inhibitors (Online Supplementary Figures S1D and S2). Harnessing the RNAseq data for expression identified significantly higher RNA levels for AURKA, slightly higher levels of $A U R K B$ with no difference in AURKC levels in TCF3-HLF ALL as compared to the other B-ALL samples (Figure 1A). Increased AURKA expression was further validated by protein expression as compared to three separate B-ALL xenograft samples (Figure 1B). Gene set enrichment analysis (GSEA) of the top 15 up-regulated Reactome pathways also emphasized cell cycle pathways (Online Supplementary Figures $S 3 A$ ) as well as pathways involving Aurora kinases in TCF3-HLF ALL (Online Supplementary Figures $S 3 B$ ). Within the class of Aurora kinase inhibitors, the AURKA selective alisertib appeared to be the most advanced in clinical testing, including pediatric dosing. ${ }^{11}$ Utilizing our functional assay, the population of B-ALL appear by and large resistant to alisertib with a median half maximal inhibitory concentration $\left(\mathrm{IC}_{50}\right)>10 \mu \mathrm{M}$ while the majority of TCF3-HLF ALL samples showed some sensitivity (Figure 1D; Online Supplementary Figures $S 1 D$ and S2). As AURKA levels can be dependent on the cell cycle we compared the levels of BIRC5 and INCENP, two proteins critical for the chromosome passenger complex during mitosis. ${ }^{12}$ Interestingly, both BIRC5 and INCENP levels were increased compared to B-ALL suggesting that these samples may have a higher population of cells progressing through the cell cycle (Figure 1C). These findings correlated with an increase in the number of cells in S phase amongst TCF3-HLF ALL and considerable sensitivity to the BIRC5 inhibitor YM155 (Figure 1D).

In order to further interrogate the effects of alisertib on TCF3-HLF ALL, HAL01 cells that carry a type 1 fusion was used as a model (Figure 2). We found that HAL01 displayed a low $\mathrm{IC}_{50}$ of $1 \mathrm{nM}$ for alisertib when assessing viability, increased apoptosis by annexin $\mathrm{V}$ staining and a significant G2/M arrest (Figure 2A to C). Treatment of these cells with alisertib appeared to disrupt the normal assembly of the spindle poles with more cells having only one spindle pole, and some cells with more than two spindle poles (Figure 2D and E). These phenotypes are similar to RNAi depletion of AURKA consistent with alisertib's effects on Aurora A kinase inhibition. ${ }^{13}$

Each TCF3-HLF ALL sample was then tested for therapeutic response in vivo in cohorts of immunodeficient NSG mice. One week after tail vein injection, cohorts were treated with vehicle, the SRC inhibitor dasatinib (40 $\mathrm{mg} / \mathrm{kg} /$ day), the BCL2 inhibitor venetoclax (25 $\mathrm{mg} / \mathrm{kg} /$ day), combination of dasatinib and venetoclax $(\mathrm{n}=5)^{14}$ or alisertib $(30 \mathrm{mg} / \mathrm{kg} / \text { day })^{15}$ by oral gavage for five days each week (Figure 3). All control animals engrafted rapidly within 2 months after injection. Only sample 3.332 and 3.310 showed significant response to dasatinib, venetoclax, or combination. Interestingly, 
A
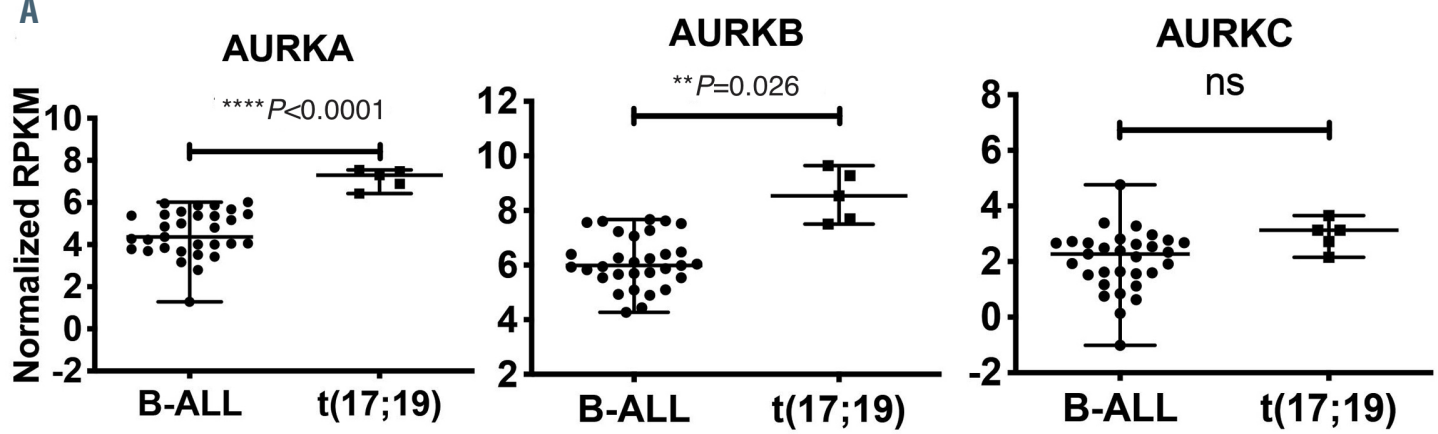

B

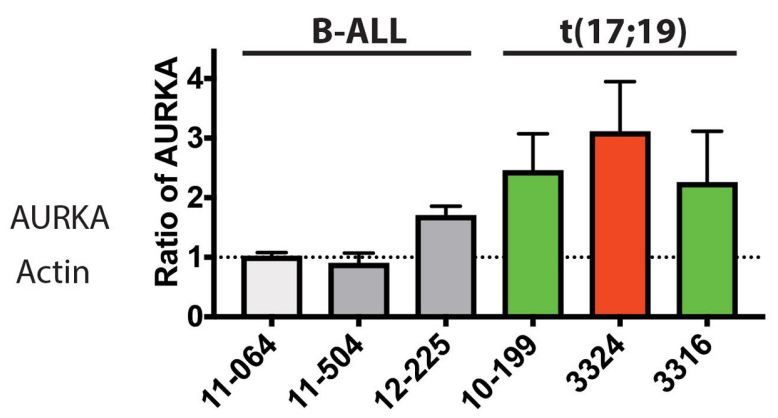

C

BIRC5

INCENP
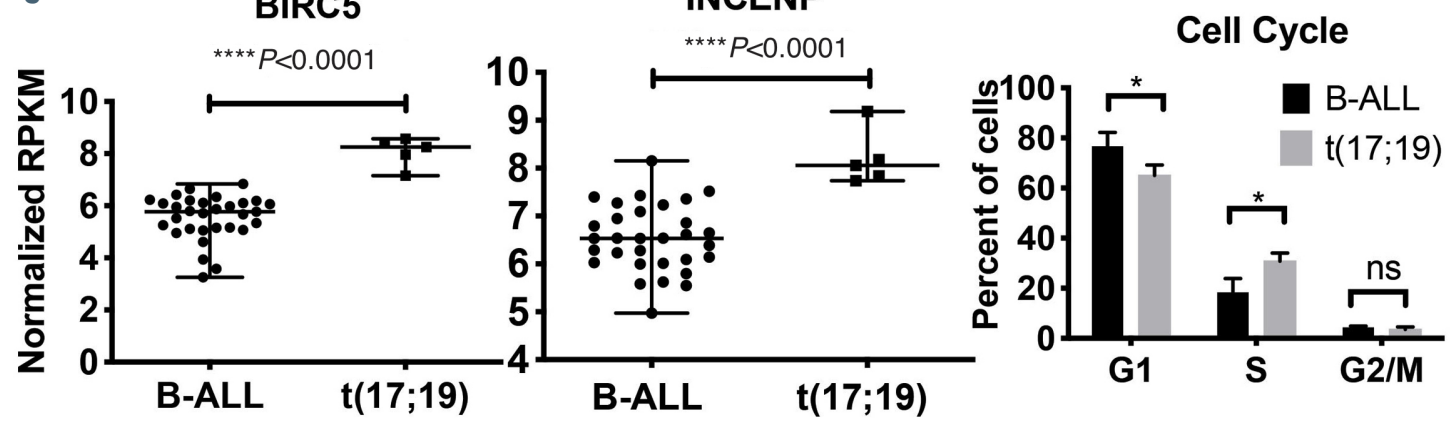

D
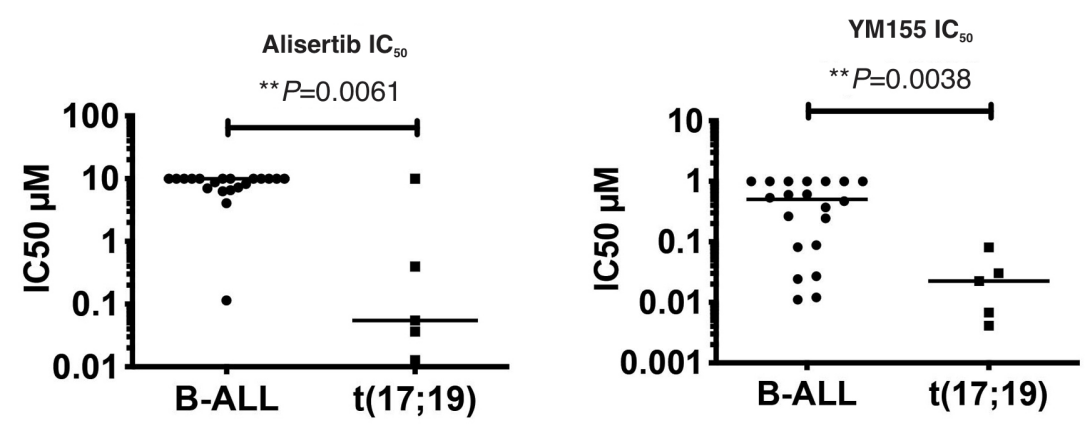

Figure 1. Sensitivity of TCF3-HLF ALL to AURKA inhibition. (A) Expression levels of AUK kinases within TCF3-HLF acute lymphoblastic leukemia (ALL) samples from RNA sequencing (RNA-seq) analysis. RPKM (reads per kilo base per million mapped reads) levels of AURKA, AURKB and AURKC were compared between $\mathrm{t}(17 ; 19)$ and remaining B-cell acute lymphoblastic leukemia (B-ALL) samples by two-tailed student's $t$-test. (B) Protein expression of $A U R K A$ in ALL. Protein extract from three primary B-ALL xenograft samples (11-064, TCF3-PBX1; 11-504, KMT2A-rearranged; 12-225, BCR-ABL1) were compared to three t(17;19) TCF3-HLF ALL xenograft samples by immunoblot (green denotes type 1 fusions, while red denotes type 2 fusions). Cell lysate was extracted and separated by standard SDS-PAGE. Protein was then transferred to PVDF membranes and immunoblotted using anti-AURKA or anti-actin. Three independent blots for each sample were quantified and AURK level for each sample was normalized to actin. Comparison of the level of AURK to each sample was normalized to the level of AURK in 11-064. Green column indicates type 1 samples, red column indicates type 2 . ANOVA $* * * P=0.0033$. (C) Expression from RNA-seq analysis comparing RPKM levels of BIRC5 and INCENP between $\mathrm{t}(17 ; 19)$ and remaining ALL samples by two-tailed student's t-test. Graphic representation of asynchronous populations within the cell cycle. Cells were fixed in $70 \%$ methanol, then stained with propidium iodide (PI), and analyzed for DNA content by flow cytometry. Comparison of the percentage of cells in G1, S and G2/M were performed from three B-ALL xenografted samples compared to three TCF3-HLF ALL samples using two-tailed student's $t$-test. (D) Half maximal inhibitory concentration $\left(\mathrm{IC}_{50}\right)$ comparison of alisertib and YM155 between $\mathrm{t}(17 ; 19)$ and remaining ALL samples by two-tailed student's $t$-test. 
these two samples do not carry the IKZF1 variant. In contrast, all animals treated with alisertib did show a significant survival advantage compared to their control counterparts.

Our results continue to confirm that TCF3-HLF ALL is a unique subset of ALL with varying degrees of response to prior published targets for therapy. In vivo validation in the murine model with our five samples suggests hetero- geneity of response to current pursued targets such as $\mathrm{BCL} 2$ and SRC. In contrast, we found significant in vivo response to the AURKA inhibitor alisertib in all of our samples tested. It is interesting that prior studies using a different set of samples showed significant in vivo response to venetoclax ${ }^{3}$ compared to our studies. This difference may be due to differences in the methodology used in the in vivo studies and/or the biology of the sam-
A

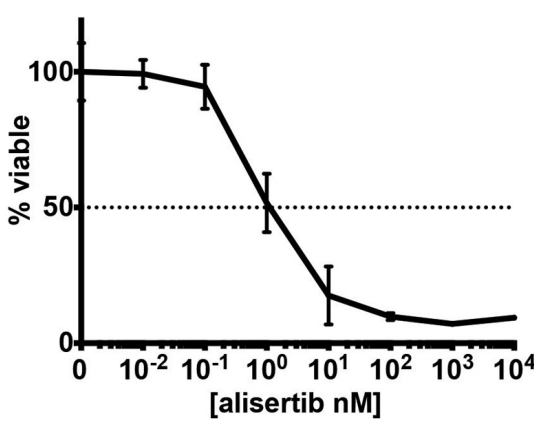

[alisertib nM]

D
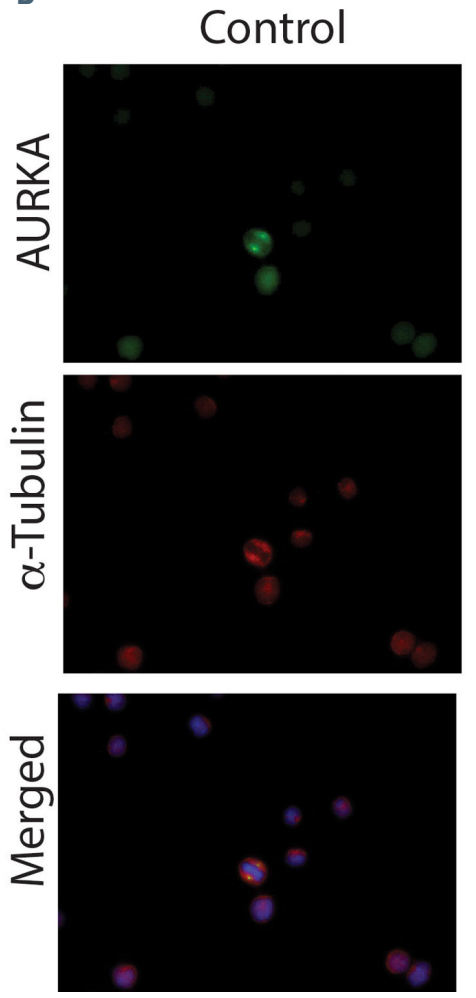

B

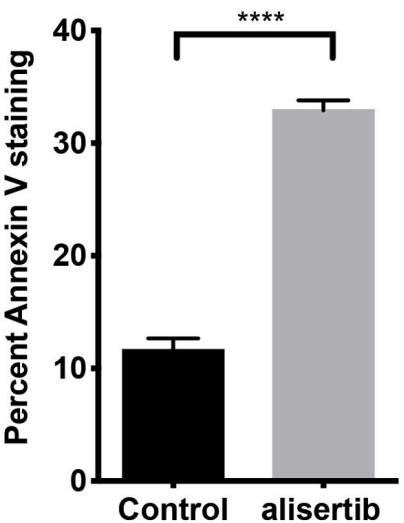

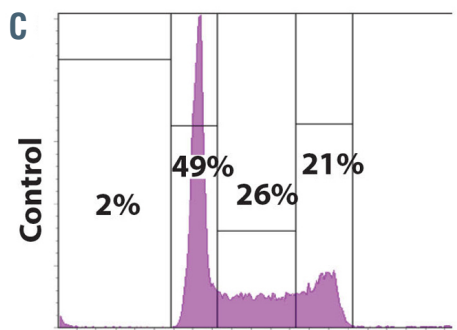

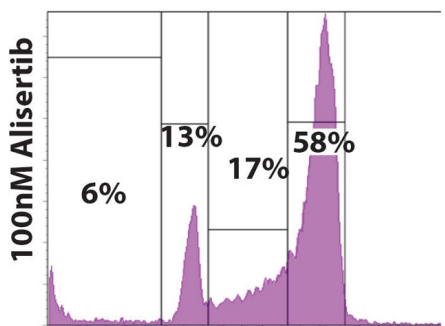

E
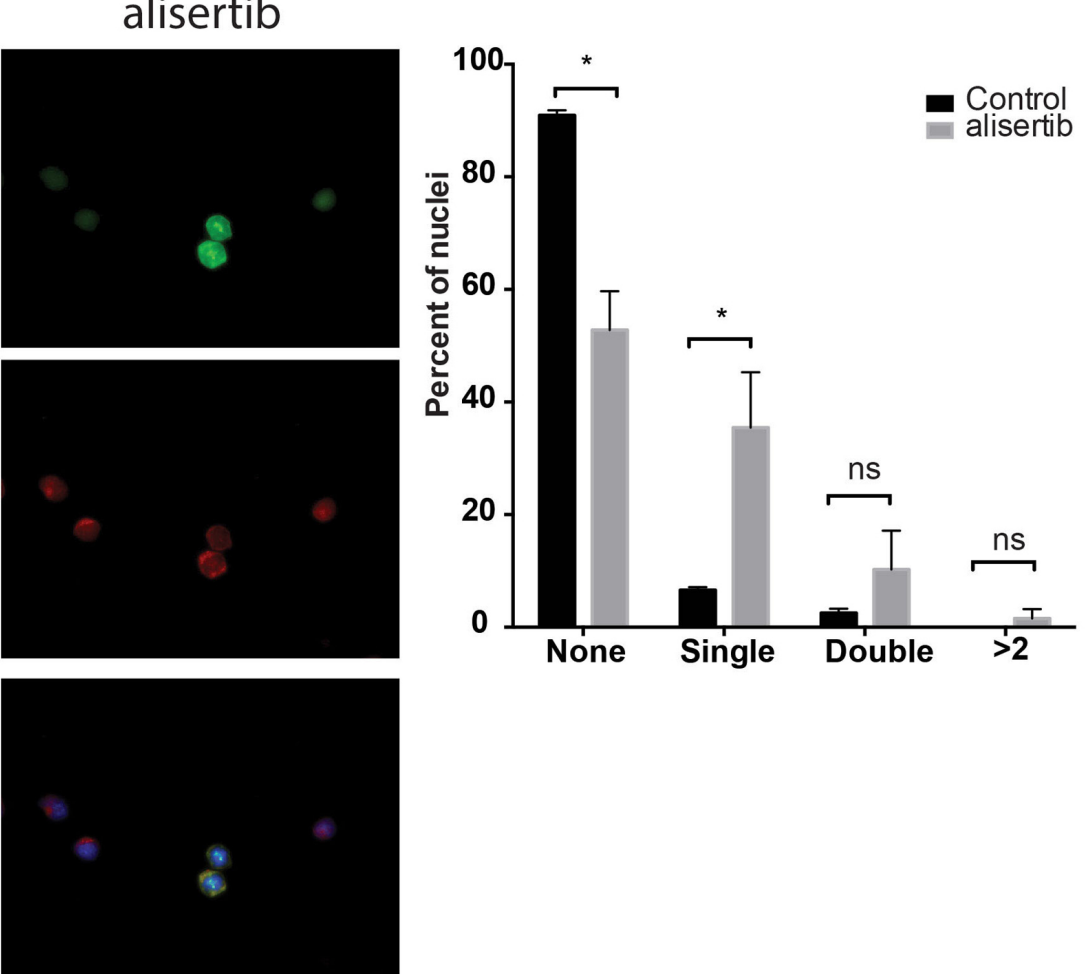

\section{E}

Figure 2. Alisertib effects on TCF3-HLF HAL01 acute lymphoblastic leukemia cells. (A) Dose-response to varying concentrations of alisertib. Cells were exposed to alisertib for 3 days then assessed for viability with MTS. Viability was normalized to untreated cells. All data points were performed in triplicate. (B) Assessment of apoptosis with annexin V staining. Cells were exposed to $100 \mathrm{nM}$ alisertib $\left(\mathrm{IC}_{90}\right)$ for 24 hours, then assessed for annexin V staining using Guava Nexin within the population of cells. All data points were performed in triplicate and compared by unpaired student's $t$-test $(* * * * P<0.0001)$. (C) $\mathrm{G} 2 / \mathrm{M}$ cell cycle arrest after exposure to alisertib. HAL01 cells were incubated with $100 \mathrm{nM}$ alisertib for 24 hours, fixed with $70 \%$ methanol and stained with propidium iodide (PI), then analyzed by flow cytometry for PI intensity. (D) Immunocytochemical fluorescence of AURKA within an asynchronous population of cells. HALO1 cells were exposed to $100 \mathrm{nM}$ alisertib for 24 hours in culture, then fixed with $4 \%$ paraformaldehyde, permeabilized with triton X-100 and stained with rabbit antiAURKA, mouse anti- $\alpha$-tubulin, secondary anti-rabbit FITC, secondary anti-mouse Texas Red, and DAPI. (E) Quantification of cells with spindle poles. Within an asynchronous population, the number of nuclei with 0 (none), 1 (single), 2 (double), or more (>2) spindle poles were counted. Each population was compared by an unpaired student's $t$-test $(* P<0.05)$. 

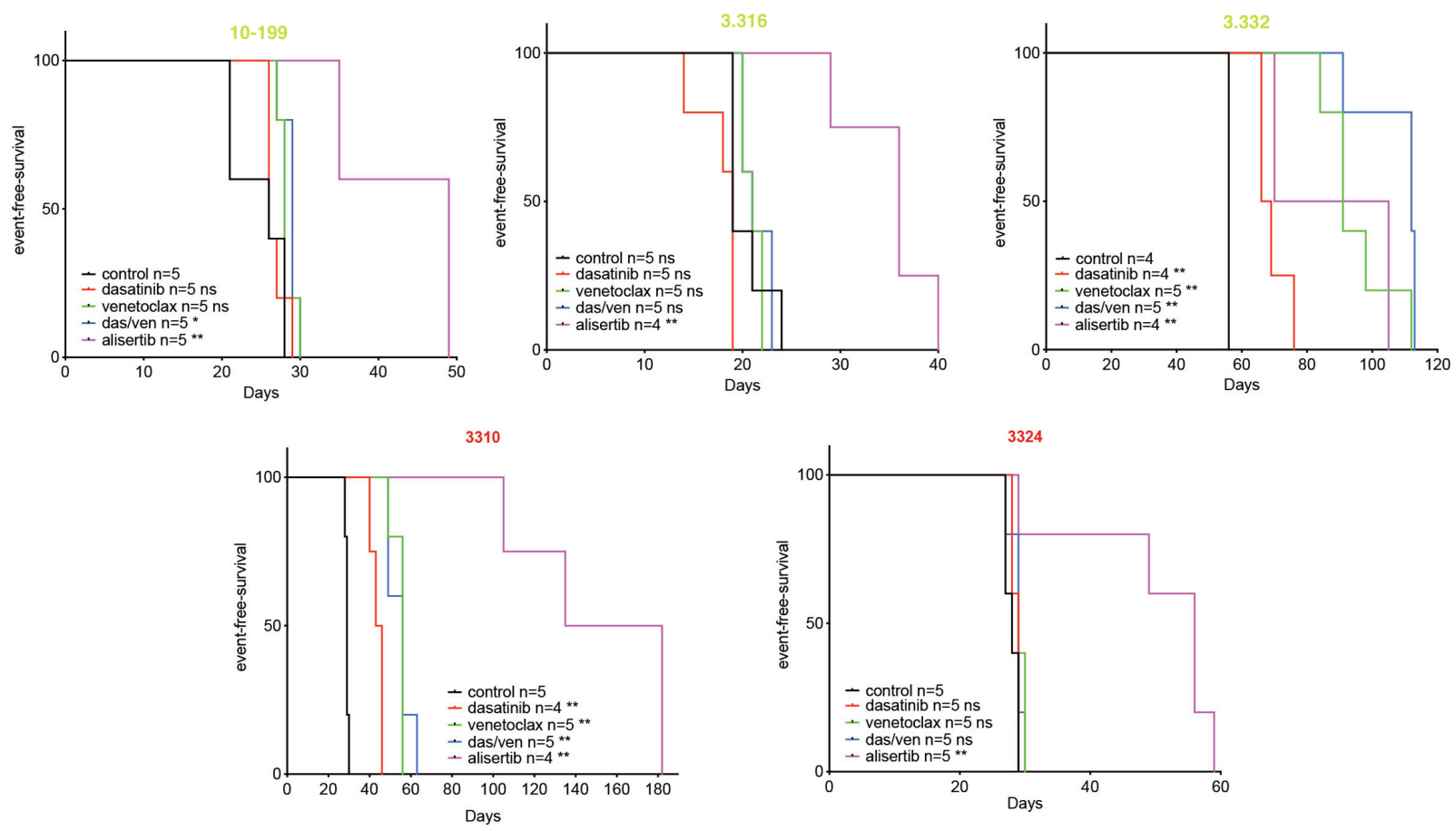

Figure 3. In vivo response of TCF3-HLF acute lymphoblastic leukemia xenografts. Kaplan-Meier plots for event-free-survival (EFS) comparing control to drug treated cohorts of five separate TCF3-HLF acute lymphoblastic leukemia (ALL) samples. Cohorts of five animals were injected with $1 \times 10^{6}$ leukemia cells per animal by tail vein. Within the first week there was some attrition due to animal loss in samples 3.316, 3.332, and 3.310 (where $n=$ the number of animals per cohort). One week after injection cohorts of four to five individuals began treatment with vehicle (control), dasatinib (40 mg/kg/day), venetoclax ( $25 \mathrm{mg} / \mathrm{kg} / \mathrm{day}$ ), combination of dasatinib (40 mg/kg/day) and venetoclax ( $25 \mathrm{mg} / \mathrm{kg} /$ day) (das/ven), or alisertib (30 mg/kg/day) by oral gavage for 5 days each week. Animals were monitored weekly for peripheral blood chimerism for human CD45 and murine CD45 by flow cytometry and daily well-being. An event was defined as peripheral blood chimerism $\geq 10 \%$ or if the animal became moribund. The top row represents type 1 TCF3-HLF rearrangements, while the bottom row represents type 2 rearrangements. Statistical comparison performed by log-rank test $\left({ }^{*} P<0.05 ; * * P<0.01\right)$.

ples within each study. Further, earlier studies did not identify AURKA as a potential target, although there appear to be similar trends with their studies of other AURK inhibitors. ${ }^{3,4}$ One very interesting observation was the sensitivity of TCF3-HLF ALL to YM155 both in our studies as well as Fischer et al. ${ }^{3}$ Unfortunately, this compound is not currently under significant clinical evaluation making the future of this drug unknown. There are also novel immunotherapeutic targets that may be pursued in future therapies including the use of the CD19 target using blinatumomab for therapeutic response followed by stem cell transplantation. ${ }^{7}$

Our studies suggest that TCF3-HLF ALL may have clinical benefit from alisertib. This compound has been and is currently under investigation for multiple different disease types. The Children's Oncology Group (COG) phase II study (clinicaltrials gov. Identifier: ADVL0921) achieved their target plasma level, but minimal objective responses were seen with significant toxicities. Their studies concurrently ran xenograft experiments with alisertib using twice daily dosing to show similar therapeutic effects, with their concern that the dose tolerated in the murine model did not reflect what is tolerated in the patient. In contrast we used $30 \mathrm{mg} / \mathrm{kg} /$ dose daily in our xenograft studies as described in Manfredi et al. ${ }^{15}$ this dose was not only well-tolerated for 21 days but had similar to improved response compared to $20 \mathrm{mg} / \mathrm{kg}$ given twice daily. These findings would suggest the dosing needed for therapeutic effect for TCF3-HLF ALL may be less in patients. Clearly our studies do not describe complete disease control with the single agent alisertib, rather a delay in disease progression as all xenograft animals eventually engrafted and succumbed to their disease. It remains unknown as to whether addition of this drug in combination therapies (e.g., conventional chemotherapy or other targeted agents such as venetoclax) may provide a beneficial response to select tumors that are dependent on AURKA. Because of the rarity of this tumor, it will take collaborative studies to test this compound in patients with TCF3-HLF ALL.

Jessica Leonard, Joelle S.J. Wolf, Michelle Degnin, ${ }^{1}$ Christopher A. Eide, ${ }^{1}$ Dorian LaTocha, ${ }^{1}$ Kyle Lenz, ${ }^{2}$ Beth Wilmot, ${ }^{3}$ Charles G. Mullighan, ${ }^{4}$ Mignon Loh, ${ }^{5}$ Stephen P, Hunger, ${ }^{6}$ Brian J. Druker, ${ }^{1,7}$ Marc M. Loriaux, ${ }^{8}$ Jeffrey W. Tyner ${ }^{1,9}$ and Bill H. Chang

${ }^{1}$ Division of Hematology and Medical Oncology, Department of Medicine and OHSU Knight Cancer Institute, OHSU, Portland, OR; ${ }^{2}$ Division of Hematology and Oncology, Department of Pediatrics and OHSU Knight Cancer Institute, OHSU, Portland, OR; ${ }^{3}$ Division of Bioinformatics and Computational Biology, Department of Medical Informatics and Clinical Epidemiology, and OHSU Knight Cancer Institute, OHSU, Portland, OR; ${ }^{4}$ Department of Pathology, St Jude Children's Research Hospital and University of Tennessee Health Science Center, Memphis, TN; ${ }^{5}$ Department of Pediatrics, Benioff Children's Hospital, Helen Diller Family Comprehensive Cancer Center, University of California, San Francisco, San Francisco, CA; ${ }^{6}$ Department of Pediatrics and Center for Childhood Cancer Research, Children's Hospital of Philadelphia and Perelman School of Medicine, University of Pennsylvania, Philadelphia, PA; ${ }^{7}$ Howard Hughes Medical Institute, Chevy Chase, MD; ${ }^{8}$ Department of Pathology and OHSU Knight Cancer Institute, OHSU, Portland, OR and ${ }^{9}$ Department of Cell, Molecular, and Cancer Biology, and OHSU Knight Cancer Institute, OHSU, Portland, OR, USA 
Correspondence:

BILL H. CHANG - changb@ohsu.edu

doi:10.3324/haematol.2021.278692

Received: March 2, 2021.

Accepted: June 21, 2021.

Pre-published: July 8, 2021.

Disclosures: CGM received research funding from AbbVie and Pfizer, speaking fees and stock from Amgen; Advisory Board Illumina; JWT received research support from Agios, Aptose, Array, AstraZeneca, Constellation, Genentech, Gilead, Incyte, Janssen, Petra, Seattle Genetics, Syros, Takeda, and Tolero; SPH has received consulting fees from Novartis, honoraria from Jazz, Amgen and Servier, and owns common stock in Amgen; BJD's potential competing interests include: SAB: Aileron Therapeutics, Therapy Architects (ALLCRON), Cepheid, Vivid Biosciences, Celgene, RUNX1 Research Program, Nemucore Medical Innovations, Novartis, Gilead Sciences (inactive), Monojul (inactive); SAB and stock: Aptose Biosciences, Blueprint Medicines, EnLiven Therapeutics, Iterion Therapeutics, GRAIL; scientific founder: MolecularMD (inactive, acquired by ICON); board of directors and stock: Amgen, Vincerx Pharma; board of directors: Burroughs Wellcome Fund, CureOne; joint steering committee: Beat AML LLS; founder: VB Therapeutics; sponsored research agreement: EnLiven Therapeutics; clinical trial funding: Novartis, Bristol-Myers Squibb, Pfizer; royalties from Patent 6958335 (Novartis exclusive license) and OHSU and Dana-Farber Cancer Institute (one Merck exclusive license and one CytoImage, Inc. exclusive license).

Contributions: JL performed the research and wrote the manuscript; $J S W$ performed the research and wrote the paper; MD analyzed the data and wrote the manuscript; CAE analyzed the data and wrote the manuscript; DL performed the flow cytometry and wrote the paper; $K L$ performed the research and wrote the paper; $B W$ contributed analytical tools, analyzed the data and wrote the manuscript; $C G M$ contributed vital reagents and wrote the manuscript; $M L$ contributed vital reagents and wrote the manuscript; SPH contributed vital reagents and wrote the manuscript; BJD contributed vital reagents and wrote the manuscript; MML contributed vital reagents and wrote the manuscript; JWT designed the research, contributed vital reagents and wrote the manuscript; $B H C$ designed the research, performed the research, analyzed the data and wrote the manuscript.

Acknowledgments: we wish to express our deepest gratitude to the patients and families that agreed to submit these precious samples to their respective biorepositories.

Funding: these studies were partially funded by the Hyundai Hope on Wheels, Newman's Own Foundation, and Tucker's Toy box, American Lebanese Syrian Associated Charities of St. Jude Children's Research Hospital, NCI P30 CA021765 and R35 CA197695.
Data sharing statement: for original data, please contact changb@ohsu.edu.

\section{References}

1. Gu Z, Churchman ML, Roberts KG, et al. PAX5-driven subtypes of B-progenitor acute lymphoblastic leukemia. Nat Genet. 2019; 51(2):296-307.

2. Glover JM, Loriaux M, Tyner JW, Druker BJ, Chang BH. In vitro sensitivity to dasatinib in lymphoblasts from a patient with $t(17 ; 19)(q 22 ; p 13)$ gene rearrangement pre-B acute lymphoblastic leukemia. Pediatr Blood Cancer. 2012;59(3):576-579.

3. Fischer U, Forster M, Rinaldi A, et al. Genomics and drug profiling of fatal TCF3-HLF-positive acute lymphoblastic leukemia identifies recurrent mutation patterns and therapeutic options. Nat Genet. 2015;47(9):1020-1029.

4. Frismantas V, Dobay MP, Rinaldi A, et al. Ex vivo drug response profiling detects recurrent sensitivity patterns in drug-resistant acute lymphoblastic leukemia. Blood. 2017;129(11):e26-e37.

5. Panagopoulos I, Micci F, Thorsen J, et al. A novel TCF3-HLF fusion transcript in acute lymphoblastic leukemia with a $\mathrm{t}(17 ; 19)$ (q22;p13). Cancer Genet. 2012;205(12):669-672.

6. Minson K, Vear S, Prasad P, Domm J, Borinstein S, Frangoul H. The prognostic significance of $t(17 ; 19)$ on outcome of children with pre$B$ acute lymphoblastic leukemia (ALL). Pediatr Blood Cancer. 2011; 56(6):927

7. Mouttet B, Vinti L, Ancliff P, et al. Durable remissions in TCF3-HLF positive acute lymphoblastic leukemia with blinatumomab and stem cell transplantation. Haematologica. 2019;104(6):e244-e247.

8. Ma X, Edmonson M, Yergeau D, et al. Rise and fall of subclones from diagnosis to relapse in pediatric B-acute lymphoblastic leukaemia. Nat Commun. 2015;6:6604.

9. Watanabe A, Inukai T, Kagami K, et al. Resistance of t(17;19)-acute lymphoblastic leukemia cell lines to multiagents in induction therapy. Cancer Med. 2019;8(11):5274-5288.

10. Tyner JW, Tognon CE, Bottomly D, et al. Functional genomic landscape of acute myeloid leukaemia. Nature. 2018;562(7728):526-531.

11. Mosse YP, Fox E, Teachey DT, et al. A phase II study of alisertib in children with recurrent/refractory solid tumors or leukemia: Children's Oncology Group Phase I and Pilot Consortium (ADVL0921). Clin Cancer Res. 2019;25(11):3229-3238.

12. Adams R, Maiato H, Earnshaw W, Carmena M. Essential roles of Drosophila inner centromere protein (INCENP) and aurora B in histone $\mathrm{H} 3$ phosphorylation, metaphase chromosome alignment, kinetochore dysjunction, and chromosome segregation. J Cell Biol. 2001; 153(4):856-880.

13. Zhou H, Kuang J, Zhong L, et al. Tumour amplified kinase STK15/BTAK induces centrosome amplification, aneuploidy and transformation. Nat Genet. 1998;20(2):189-193.

14. Leonard JT, Rowley JS, Eide CA, et al. Targeting BCL-2 and ABL/LYN in Philadelphia chromosome-positive acute lymphoblastic leukemia. Sci Transl Med. 2016;8(354):354ra114.

15. Manfredi MG, Ecsedy JA, Chakravarty A, et al. Characterization of Alisertib (MLN8237), an investigational small-molecule inhibitor of aurora A kinase using novel in vivo pharmacodynamic assays. Clin Cancer Res. 2011;17(24):7614-7624. 